\title{
In utero exposure to extra vitamin D from food fortification and the risk of subsequent development of gestational diabetes: the D-tect study
}

Amélie Keller ${ }^{1^{*}}$ (D) Maria Stougård ${ }^{1}$, Peder Frederiksen ${ }^{1}$, Fanney Thorsteinsdottir ${ }^{1}$, Allan Vaag ${ }^{2}$, Peter Damm ${ }^{3,4}$, Ramune Jacobsen ${ }^{1,5}$ and Berit L. Heitmann ${ }^{1,6,7,8}$

\begin{abstract}
Background: The primary aim of this study was to assess whether exposure during fetal life to extra vitamin D from food fortification was associated with a reduction in the risk of subsequently developing gestational diabetes mellitus (GDM). Furthermore, we examined whether the effect of the vitamin D from fortification differed by women's season of birth.

Methods: This semi-ecological study is based on the cancellation in 1985 of the mandatory policy to fortify margarine with vitamin $D$ in Denmark, with inclusion of entire national adjacent birth cohorts either exposed or unexposed to extra vitamin D in utero. The identification of GDM cases later in life among both exposure groups was based on the Danish national health registers. Logistic regression analyses generating odds ratios (ORs) and 95\% confidence intervals (95\% Cls) were performed.
\end{abstract}

Results: Women who were prenatally exposed to the extra vitamin $D$ from fortification tended to have a lower risk of subsequently developing GDM than unexposed women (OR $0.87,95 \% \mathrm{Cl} 0.74,1.02, P=0.08)$. When analyses were stratified by women's season of birth, exposed women born in spring had a lower risk of developing GDM compared to unexposed subjects (OR 0.68, 95\% Cl 0.50,0.94, $p=0.02$ ).

Conclusion: This study suggests that prenatal exposure to extra vitamin $D$ from mandatory fortification may lower the risk of developing gestational diabetes among spring-born women.

Trial registration: This study is part of the D-tect project, which is registered on clinicaltrials.gov: NCT03330301.

Keywords: Vitamin D, Gestational diabetes mellitus, Public health epidemiology, Fetal programming, Food intake

\section{Background}

Gestational diabetes mellitus (GDM) is defined as "carbohydrate intolerance of varying severity with onset or first recognition during pregnancy" [1]. In Europe, GDM is one of the most common pregnancy disorders with an estimated prevalence of 2-6\% [2]. Women who develop GDM are more likely to experience pregnancy

\footnotetext{
* Correspondence: amelie.cleo.keller@regionh.dk

${ }^{1}$ Research Unit for Dietary Studies at The Parker Institute Bispebjerg and Frederiksberg Hospital, part of the Copenhagen University Hospital - The capital Region, Nordre Fasanvej 57, vej 8, entrance 11, 2000 Frederiksberg, Denmark

Full list of author information is available at the end of the article
}

related complications such as pregnancy induced hypertension, obstructed labor and macrosomic newborns (birth weight $>4000 \mathrm{~g}$ ) and have a high risk of later developing type 2 diabetes (T2D) [3]. Furthermore, offspring of GDM mothers are at increased risk of metabolic syndrome, GDM, T2D, obesity and cardiovascular disease later in life [2].

Obesity, increased maternal age, family history of T2D and previous delivery of a macrosomic infant are all known risk factors for GDM [4]. Nutritional risk factors such as high red and processed meat consumption as well as low-fiber and high glycemic index diets may increase the risk of GDM $[5,6]$. In regards to the role of

(c) The Author(s). 2018 Open Access This article is distributed under the terms of the Creative Commons Attribution 4.0 International License (http://creativecommons.org/licenses/by/4.0/), which permits unrestricted use, distribution, and 
vitamin D on the risk of GDM development, discrepant evidence is present in the literature $[7,8]$, however recent evidence suggests that low blood vitamin D level during pregnancy could increase the risk of GDM, and vitamin D supplementation during pregnancy could improve GDM condition [9]. Vitamin D is a fat-soluble vitamin and a secosteroid obtained either from the diet (as $D_{2}$ from vegetables and $D_{3}$ from food such as oily fish and dairy products or fortified food and supplements); or through subcutaneous synthesis by exposure to sunlight [10]. The presence of nuclear vitamin D receptors and the vitamin D-activating 1- $\alpha$-hydroxylase enzyme in the placenta suggests that vitamin D plays an important role during pregnancy [4] and may influence fetal development. Moreover, studies have also shown that low fetal or infancy vitamin D may influence later risk of disease development, such as type 1 diabetes (T1D) and pre-eclampsia, via so called early programming $[11,12]$. However, no previous studies have examined if women with a lower fetal vitamin D exposure may be at higher subsequent risk of developing GDM than women with a higher fetal exposure to vitamin D.

In Denmark, fortification of margarine with vitamin A and D started in 1937 [13], and fortification with vitamin D was stopped by law on the 1st June 1985 [12, 14]. Between 1962 and 1985, margarine was fortified with $1.25 \mu \mathrm{g}$ vitamin $\mathrm{D} / 100 \mathrm{~g}$ of margarine, representing up to $29 \%$ of total dietary vitamin D intake (average 13\%) [15]. To assess the association between exposure to extra vitamin $\mathrm{D}$ from food fortification in utero and the subsequent risk of developing GDM, all women born in Denmark two years prior and two years after the termination of the vitamin $\mathrm{D}$ fortification were included in this study. The primary aim of this study was to assess whether exposure during fetal life to extra vitamin D from food fortification was associated with a decreased risk of subsequently developing GDM. Furthermore, we examined whether the effect of the margarine fortification differed by women's season of birth.

\section{Method}

\section{Data sources}

The Danish Civil Registration System (CRS) was used to retrieve information about the study population. Since April 1968, all people living in Denmark are registered by a 10-digit civil person registration (CPR) number in the CRS. Using this number, linkage of individual information from different registers and databases was possible. Information about antenatal care visits for all women with permanent residence in Denmark has been registered in the Danish Medical Birth Register (DMBR) since 1973 [16]. Since 1977, all non-psychiatric hospitals' discharges, and since 1995, all emergency and outpatient departments' discharges have been registered in the
Danish National Patient Register (DNPR) [17]. Thus, using the CPR, information about pregnancy and offspring characteristics were retrieved from the DMBR, and the DNPR was used to identify diagnoses of GDM using the International Classification of Diseases (ICD) 10 code 024.4 .

\section{Study population}

All women born in Denmark around the fortification termination, between June 1983 and August 1988 and who later gave birth were included in this study. Based on the termination of the margarine fortification policy on the 1st June 1985, women born between June 1983 and May 1985 were considered to have been prenatally exposed to extra vitamin D. To ensure the unexposed group was truly unexposed, a 15-month wash-out period was introduced, from June 1985 to September 1986, accounting for the 9 months of pregnancy and an additional 6 months to ensure vitamin D fortified margarine was no longer available on the Danish market or households. Thus, women born between September 1986 and August 1988 were considered to be unexposed (Fig.1).

The main analyses were restricted to nulliparous women who gave birth for the first time to a singleton infant between January 2004 and February 2016 after at least 22 weeks of gestation. In addition, women had to be between 20.6 and 27.5 years of age at time of delivery, to ensure same length of follow-up in both exposure groups. Therefore, women were excluded from the analyses if they had missing information on the following variables: age at delivery, whether it was a singleton or multiple births and offspring's gestational age at delivery. Furthermore, women who were born during the 15-month's wash-out period were excluded. Women with a pre-pregnancy BMI $\leq 15$ or $\geq 50 \mathrm{~kg} / \mathrm{m}^{2}$ were also excluded as erroneous data-entry in the DMBR was likely. Women whose own birth weight was registered as $0 \mathrm{~g}$ in the DMBR were also excluded as this likely reflected erroneous data entry in the DMBR.

\section{Outcome}

In 2003, new national recommendations for screening and treatment of GDM, were implemented which improved GDM detection and management [18]. Since, selective screening for GDM has been recommended in Denmark to women with at least one of the following risk factors for GDM: previous GDM, previous birth to a large child (birth weight $\geq 4500 \mathrm{~g}$ ), a pre-pregnancy body mass index (BMI) $\geq 27 \mathrm{~kg} / \mathrm{m}^{2}$, a family history of diabetes, and glucosuria [18]. A $75 \mathrm{~g} 2$-h oral glucose tolerance test (OGTT) is performed at 27-30 weeks and also in early pregnancy (14-20 weeks) for women with glucosuria, previous GDM or more than one of the aforementioned risk factors. The diagnostic criteria for GDM are a 2-h capillary blood or venous plasma glucose 


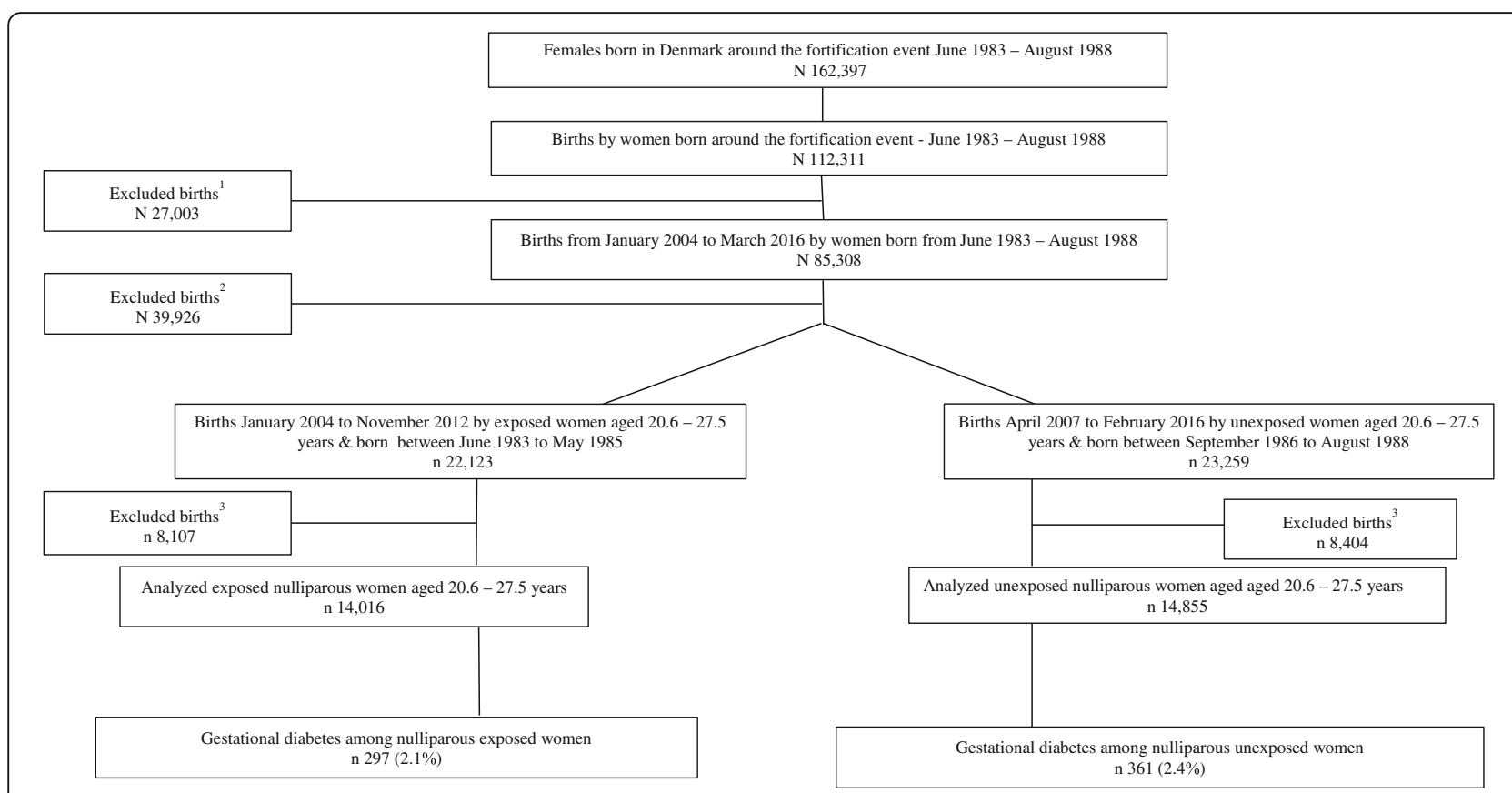

Fig. 1 Flow chart of the study population, 'Exclusion criteria: Women born during the 15 months' wash-out period from June 1985 to August 1986. ${ }^{2}$ Exclusion criteria: Women $<20.6$ and $>27.5$ years old or missing. ${ }^{3}$ Exclusion criteria: gestational weeks was $<22$ weeks or missing, pre-pregnancy BMI was $<15 \mathrm{~kg} / \mathrm{m}^{2}$ or $>50 \mathrm{~kg} / \mathrm{m} 2$, women's own birth weight was $0 \mathrm{~g}$, multiple births, stillbirths, multiparous women

$\geq 9 \mathrm{mmol} / \mathrm{L}$ [18]. To ensure GDM diagnosis was harmonized all over Denmark, GDM diagnoses from 1st January 2004 and onwards were included in this study.

\section{Covariates}

Information on smoking status during pregnancy, age at time of delivery, birth weight, pre-pregnancy BMI, singleton and multiple births, offspring gender, gestational age and parity were retrieved from the DMBR. Since 1997, data on smoking habits of pregnant women were collected in the DMBR. Smoking status during pregnancy was categorized into current smokers, former smokers (women who stopped smoking during or after the first trimester in the current pregnancy) and non-smokers. Pre-pregnancy BMI, based on self-reported height and weight at the first antenatal visit, was included in the DMBR since 2004. Pre-pregnancy BMI was categorized into the four following categories: underweight $<18.5 \mathrm{~kg} / \mathrm{m}^{2}$, normal weight $18.5-$ $24.9 \mathrm{~kg} / \mathrm{m}^{2}$, overweight $25.0-29.9 \mathrm{~kg} / \mathrm{m}^{2}$, obese $\geq 30 \mathrm{~kg} / \mathrm{m}^{2}$. Women's birth weight was categorized as follow: $<2500 \mathrm{~g}$; 2500-4000 g; > $4000 \mathrm{~g}$ [19]. Offspring's gestational age at delivery was categorized based on weeks + days as follow: $<37,37+0$ to $37+6,38+0$ to $38+6,39+0$ to $39+6,40$ +0 to $40+6,41+0$ to $41+6$ and $\geq 42$ complete weeks of gestation [12]. Furthermore, size for gestational age (small for gestational age (SGA) (birth weight $<10$ th centile for gestational age and gender) and large for gestational age (LGA) (birth weight $>$ 90th centile for gestational age and gender)) using Danish references was assessed [20].
Seasonality of birth in exposed/unexposed women was defined according to month of birth: Winter-born: November through January; Spring-born: February through April; Summer-born: May through July; Autumn-born: August through October. This categorization was based on the seasonal variation in serum $25(\mathrm{OH}) \mathrm{D}$ concentration among individuals from countries in northern latitudes [21].

\section{Statistical analyses}

This study used entire birth cohorts of all women who were born in adjacent years around the termination of margarine fortification with vitamin $\mathrm{D}$. As the women were unselected and, for their age, generally representative of the Danish population of women, in relation to both exposure status and development of GDM, we hypothesized that adjustment for covariates was not necessary. To confirm this hypothesis, a causal diagram, the directed acyclic graph (DAG), was used to identify possible confounders and to build the statistical regression analyses models [22]. Year of birth (as a proxy for exposure to vitamin D fortified margarine) was put into the model as the exposure of interest and GDM was the outcome variable of interest. The covariates mentioned above were put into the models as potential confounders. The presence or absence of a direct association between each variable-pair was assessed using a scientific framework built on theoretical and biological evidences from the literature [23]. This stepwise approach was used to draw a DAG on dagitty.net 
(Online-Only Additional file 1: DAG), from which the following statistical models were developed:

- Crude model: no adjustment

- Model 1: adjustment for women's season of birth

Chi-squared test was used to test the differences between exposed and unexposed women for categorical variables and Mann-Whitney rank-sum test for skewed continuous data. Test for normality was not performed for age distribution, as it was a priori skewed because of the age restriction applied. The association between fetal exposure to vitamin $\mathrm{D}$ fortified margarine and the risk of developing GDM was examined by logistic regression generating odds ratios (ORs) and 95\% confidence intervals (95\% CIs). The effect of the vitamin D fortification on the fetus was expected to have most effect when stores are low, such as winter pregnancies; therefore, to evaluate whether women's season of birth modified the association between prenatal vitamin D exposure and GDM, stratified analyses by women's season of birth were performed.

\section{Sensitivity analyses}

The prevalence of GDM has increased over the years and is higher at increased maternal age [24]. Therefore, trends in GDM incidence by maternal age, women's year of birth and offspring's year of birth were tested using Chi-squared test.

Prevalence of overweight and obesity among women of childbearing age, smoking habits during pregnancy and age at delivery have changed over time, and thus might have been different between exposed and unexposed women in this study. Therefore, sensitivity analyses adjusted for women's pre-pregnancy BMI, smoking status in pregnancy and age at delivery were performed (Online-Only Additional file 1: Tables S1-S3).

The amount of bright sunshine hours during pregnancy has previously been reported to be an important variable to be adjusted for while analyzing exposure-outcome association in a similar setting [11], therefore, sensitivity analyses adjusted for total and trimestral gestational sunshine hours were performed. The data on bright sunshine hours were obtained from the Danish Meteorological Institute (DMI) [25]. Summary monthly sunshine hours during the nine months prior to the month of birth, bounded to each individual were calculated. First, second and third trimester sunshine hours were also calculated by summing recoded monthly sunshine hours during 1st, 2nd and 3rd thirds of these 9 months, respectively [11] (Online-Only Additional file 1: Tables S4a-S4 h).

Women born during the 15-month wash-out period were gradually less exposed to vitamin D from the fortified margarine. To test the additional hypothesis that these women have a higher risk of developing GDM than the exposed women, and a lower risk than the unexposed women, analyses with the three exposure groups were additionally performed (Online-Only Additional file 1: Tables S5a -S5b).

The statistical software package Stata 13 (StataCorp LP, College Station, Texas, 2013; https://www.stata.com/) was used for all data management and analyses. A level of significance as defined through $p$-values $p<0.05$ was used.

\section{Results}

There were $297(2.1 \%)$ cases of GDM among women who were prenatally exposed to vitamin $\mathrm{D}$ fortified margarine $(N=14,016)$ compared to $361(2.4 \%)$ GDM cases among the unexposed $(\mathrm{N}=14,855)(p=0.08)$ (Fig. 1). Maternal age at delivery was statistically slightly higher but the difference being clinically not relevant among the exposed compared to the unexposed with a median $(5 ; 95$ percentiles) of $25.2(21.4 ; 27.3)$ and $25.1(21.2 ; 27.3)$ years, respectively $(p<0.0001)$. Unexposed women were more likely to be former smokers and non-smokers compared to exposed women (5.6\% vs. $5.2 \%$ and $81.3 \%$ vs.79.1\%, respectively). Exposed women more often gave birth after 42 weeks of gestation (6.0\% vs. $3.4 \%)$, and slightly more often had a pre-pregnancy BMI between 18.5 and $25 \mathrm{~kg} / \mathrm{m}^{2}$ than unexposed women $(60.7 \%$ vs. $59.6 \%)(p=0.01)$. There was no difference in the distribution of the women's season of birth between the two exposure groups $(p=0.8)$. Women born between 1983 and 1985 were exposed to more hours of bright sunshine during gestation than women born after the vitamin $\mathrm{D}$ fortification termination, median (5; 95 percentiles) $1108(752 ; 1311)$ and $1004(772 ; 1320)$ hours respectively $(p<0.0001)$ (Table 1$)$.

Women prenatally exposed to the vitamin $\mathrm{D}$ fortification tended to have a lower risk of developing GDM than unexposed women (Crude model: OR 0.87, 95\%CI $0.74,1.02, p=0.08$; Model 1: OR 0.87, 95\%CI 0.74,1.02, $P=$ 0.08) (Table 2). Further adjustment for women born SGA or LGA to account for differences in birth weight adjusted for gestational age gave essentially similar results (data not shown). When analyses were stratified by women's season of birth, exposed women born in spring (winter pregnancies) had a lower risk of developing GDM compared to those unexposed to extra vitamin D (OR $0.68,95 \% \mathrm{CI}$ $0.50,0.94, p=0.02$ ) (Table 3).

Results from sensitivity analyses were essentially similar to the main results (Online-Only Additional file 1: Tables S1-S4). Women born during the wash-out period tended to have a higher risk of developing GDM compared to exposed women (Crude model: OR 1.18, 95\%CI 0.99,1.40, $p=0.07$; Model 1: OR 1.17, 95\%CI $0.98,1.40, \mathrm{p}=0.08)$ and a similar risk compared to the unexposed women (Crude model: OR 1.02, 95\%CI $0.87,1.21, p=0.78$; Model 1: OR 1.02, 95\%CI 0.86,1.21, $p=$ 0.83 ), however the differences were not significant (Online-Only Additional file 1: Tables S5a-S5b). 
Table 1 Characteristics of women prenatally exposed and unexposed to vitamin D fortified margarine and their offspring

\begin{tabular}{|c|c|c|c|c|c|}
\hline & \multicolumn{2}{|c|}{ Exposed } & \multicolumn{2}{|c|}{ Unexposed } & \multirow[t]{2}{*}{$p$-value } \\
\hline & $\mathrm{N}$ & Median (5,95 percentiles) & $\mathrm{N}$ & Median (5,95 percentiles) & \\
\hline Age at delivery ${ }^{\mathrm{a}, \mathrm{c}}$ & 14,016 & $25.2(21.4 ; 27.3)$ & 14,855 & $25.1(21.2 ; 27.3)$ & $<0.0001$ \\
\hline Gestational sunshine hours ${ }^{\mathrm{a}, \mathrm{c}, \mathrm{e}}$ & 14,016 & $1108(752 ; 1311)$ & 14,855 & $1004(772 ; 1320)$ & $<0.0001$ \\
\hline \multirow[t]{2}{*}{ Offspring's birth weight $(g)^{b, d}$} & 14,016 & $3460(2572 ; 4270)$ & 14,855 & $3455(2560 ; 4260)$ & 0.3 \\
\hline & & Percentage & & Percentage & \\
\hline$G$ stational diabetes mellitus ${ }^{a}$ & 297 & 2.1 & 361 & 2.4 & 0.08 \\
\hline Pre-pregnancy $B M l^{a, d}$ & & & & & 0.01 \\
\hline$<18.5 \mathrm{~kg} / \mathrm{m}^{2}$ & 636 & 4.5 & 800 & 5.4 & \\
\hline $18.5-24.9 \mathrm{~kg} / \mathrm{m}^{2}$ & 8514 & 60.7 & 8860 & 59.6 & \\
\hline $25-29.9 \mathrm{~kg} / \mathrm{m}^{2}$ & 2984 & 21.3 & 3154 & 21.2 & \\
\hline$\geq 30 \mathrm{~kg} / \mathrm{m}^{2}$ & 1882 & 13.4 & 2041 & 13.7 & \\
\hline Size for gestational age ${ }^{a}$ & & & & & 0.7 \\
\hline SGA & 1741 & 12.4 & 1891 & 12.7 & \\
\hline NGA & 11,299 & 80.7 & 11,961 & 80.6 & \\
\hline LGA & 955 & 6.8 & 990 & 6.7 & \\
\hline Birth weight $(g)^{a, d}$ & & & & & 0.8 \\
\hline$<2500$ & 570 & 4.1 & 593 & 4.0 & \\
\hline $2500-4000$ & 11,536 & 82.3 & 12,274 & 82.6 & \\
\hline$\geq 4000$ & 1910 & 13.6 & 1988 & 13.4 & \\
\hline Smoking status during pregnancy ${ }^{\mathrm{a}, \mathrm{d}}$ & & & & & $<0.0001$ \\
\hline Current smoker & 2170 & 15.8 & 1911 & 13.0 & \\
\hline Former smoker & 715 & 5.2 & 827 & 5.6 & \\
\hline Non-smoker & 10,890 & 79.1 & 11,935 & 81.3 & \\
\hline Season of birth ${ }^{d}$ & & & & & 0.8 \\
\hline Winter & 3214 & 23.0 & 3465 & 23.3 & \\
\hline Spring & 3568 & 25.5 & 3722 & 25.1 & \\
\hline Summer & 3675 & 26.2 & 3875 & 26.1 & \\
\hline Autumn & 3559 & 25.4 & 3793 & 25.5 & \\
\hline Offspring's gestational age at delivery (weeks) ${ }^{\mathrm{b}, \mathrm{d}}$ & & & & & $<0.0001$ \\
\hline$<37$ & 871 & 6.2 & 892 & 6.0 & \\
\hline $37+0-37+6$ & 699 & 5.0 & 727 & 4.9 & \\
\hline $38+0-38+6$ & 1688 & 12.0 & 1751 & 11.8 & \\
\hline $39+0-39+6$ & 3068 & 21.9 & 3150 & 21.2 & \\
\hline $40+0-40+6$ & 4033 & 28.8 & 4423 & 29.8 & \\
\hline $41+0-41+6$ & 2819 & 20.1 & 3414 & 23.0 & \\
\hline$\geq 42$ & 838 & 6.0 & 498 & 3.4 & \\
\hline Offspring's gender ${ }^{\mathrm{b}, \mathrm{d}}$ & & & & & 0.02 \\
\hline Female & 6920 & 49.4 & 7128 & 48.0 & \\
\hline Male & 7096 & 50.6 & 7727 & 52.0 & \\
\hline
\end{tabular}

${ }^{\mathrm{a}}$ Characteristics of women prenatally exposed and unexposed to vitamin $\mathrm{D}$ fortified margarine and ${ }^{\mathrm{b}}$ their offspring ctested by Mann-Whitney rank-sum test and ${ }^{d}$ tested by $\mathrm{X}^{2}$-test

eSummary monthly sunshine hours during the nine months prior to the month of birth, bounded to each individual

There was a higher GDM incidence with higher age at delivery $(p=0.004)$, and both women's $(p=0.04)$ and offspring's years of birth $(p<0.0001)$.

\section{Discussion}

This study suggests that prenatal exposure to extra vitamin $\mathrm{D}$ from mandatory fortification may lower the 
Table 2 Crude and adjusted odds ratio (OR) and 95\% confidence intervals $(95 \% \mathrm{Cl})$ for gestational diabetes mellitus among women prenatally exposed and unexposed to vitamin D fortified margarine

\begin{tabular}{|c|c|c|c|c|c|c|c|}
\hline \multirow[t]{2}{*}{ Exposure } & \multirow[b]{2}{*}{$\mathrm{N}$} & \multicolumn{3}{|c|}{ Crude $^{a}$} & \multicolumn{3}{|c|}{ Model $1^{a}$} \\
\hline & & $\overline{O R}$ & $95 \% \mathrm{Cl}$ & $\overline{p \text {-value }}$ & $\overline{O R}$ & $95 \% \mathrm{Cl}$ & $p$-value \\
\hline Unexposed & 14,855 & 1 & & & 1 & & \\
\hline Exposed & 14,016 & 0.87 & $0.74,1.02$ & 0.08 & 0.87 & $0.74,1.02$ & 0.08 \\
\hline
\end{tabular}

${ }^{a}$ Crude model: no adjustment; Model 1a: adjustment for women's season of birth

risk of developing gestational diabetes among spring-born women. This finding suggests that the extra vitamin $\mathrm{D}$ from fortification may have been of particular benefit to offspring of mothers who were pregnant during most of the dark months when vitamin D skin synthesis is low or null, and when diet or supplementation are the sole sources of vitamin D.

Few previous studies have examined association between early exposure to vitamin D and the development of diabetes later in life. One previous longitudinal study found an association between low concentrations of $25(\mathrm{OH}) \mathrm{D}$ in pregnant mothers and fasting insulin concentrations and insulin resistance in their children at 9.5 years of age [26]. As glucose homeostasis variables tend to track from childhood to adulthood [27], $25(\mathrm{OH}) \mathrm{D}$ concentration during fetal life might also influence glucose homeostasis variables in childhood and may predict the development of diabetes in adulthood. In regards to T1D, a meta-analysis has concluded that vitamin $\mathrm{D}$ supplementation in early childhood may be associated with a lower risk of developing of T1D [28]; however, the risk of developing T1D was not different for subjects exposed and unexposed to extra vitamin $\mathrm{D}$ from fortified margarine in our previous study [29]. The discrepant findings

Table 3 Crude odds ratio (OR) and 95\% confidence intervals $(95 \% \mathrm{Cl})$ for gestational diabetes mellitus among women prenatally exposed and unexposed to vitamin D fortified margarine stratified by women's season of birth

\begin{tabular}{|c|c|c|c|c|c|c|}
\hline \multirow{2}{*}{$\begin{array}{l}\text { Seasons } \\
\text { of birth }\end{array}$} & \multirow[b]{2}{*}{ Exposure } & \multirow[t]{2}{*}{$N$} & \multirow[t]{2}{*}{$\%$ GDM } & \multicolumn{3}{|c|}{ Crude } \\
\hline & & & & $\overline{\mathrm{OR}}$ & $95 \% \mathrm{Cl}$ & $p$-value \\
\hline \multirow[t]{2}{*}{ Winter } & Unexposed & 3465 & 2.3 & 1 & & \\
\hline & Exposed & 3214 & 2.3 & 0.98 & $0.71,1.35$ & 0.92 \\
\hline \multirow[t]{2}{*}{ Spring } & Unexposed & 3722 & 2.6 & 1 & & \\
\hline & Exposed & 3568 & 1.8 & 0.68 & $0.50,0.94$ & 0.02 \\
\hline \multirow[t]{2}{*}{ Summer } & Unexposed & 3875 & 2.5 & 1 & & \\
\hline & Exposed & 3675 & 2.0 & 0.83 & $0.61,1.13$ & 0.23 \\
\hline \multirow[t]{2}{*}{ Autumn } & Unexposed & 3793 & 2.4 & 1 & & \\
\hline & Exposed & 3559 & 2.4 & 1.02 & $0.75,1.38$ & 0.91 \\
\hline
\end{tabular}

aWinter: November to January; Spring: February to April; Summer: May to July; Autumn: August to October between exposure to vitamin $\mathrm{D}$ fortification during fetal life on the risk of developing T1D and GDM might be due to a possible different genetic-environmental risk factors ratio between the two diseases; GDM being more susceptible to lifestyle related risk factors than T1D.

Several potential mechanisms could explain our finding of a potential protective effect of exposure to vitamin $\mathrm{D}$ in early life on the subsequent development of GDM. Multiple factors affecting early growth may induce changes in the structure and function of certain organs and tissues such as the pancreas [30]. Results from animal models suggest that maternal vitamin $\mathrm{D}$ restriction may lead to insulin dysregulation inducing a compensatory increase in pancreatic beta-cell mass in the offspring [30]. Another mechanism might be related to the mediating role of vitamin $\mathrm{D}$ on the association between amino acids supply and fetal growth [31]. Animal models suggest that maternal protein restriction may cause chronic disease in adulthood among first and second offspring generations [30]. Already in 1992, Hales \& Barker stated that $\beta$-cell growth and development as well as insulin secretion until late fetal life are controlled by amino acids supply [32]. Therefore, an unanswered question is whether vitamin D may be involved in the amino acids supply to the fetus. A recent human study, suggested that placental amino acid transport may be regulated by maternal vitamin $\mathrm{D}$ and vitamin $\mathrm{D}$-binding protein [33]. Hence, exposure to extra vitamin D during fetal life may promote adequate $\beta$-cell growth by mediating placental amino acid transport and decrease the risk of developing diabetes in later life [33]. Moreover, vitamin D supply might be especially important during the second gestational trimesters as animal studies have confirmed that it is an important period for development and biochemical maturation of $\beta$-cells, however these findings were not confirmed in a human study [34].

There is increasing evidence for a potential role of inflammation in the pathogenesis of T2D and GDM [35]. During pregnancy, vitamin $\mathrm{D}$ receptors and $1,25(\mathrm{OH})_{2} \mathrm{D}_{3}$ have been described to play a role in the adaptive and innate immune system as well as in the secretion of insulin by the pancreatic $\beta$-cells [36]. Therefore, exposure to extra gestational vitamin D might reduce the risk of GDM by modulating the innate immune system, particularly when vitamin D skin synthesis is low or absent. From 1937 to 1985, margarine was fortified with both vitamins D and A; and from 1985 only with vitamin A [12]. Therefore, the potential protective effect of in utero exposure to vitamin $\mathrm{D}$ fortified margarine on the development of GDM seen in this study may be due to additive, synergistic or antagonist interactions between vitamins $\mathrm{A}$ and $\mathrm{D}$. It has been reported that both vitamins may be involved in the development and functioning of human fetal pancreas. However, more studies are needed to explain these 
mechanisms and their potential role in the development of diabetes in humans [14].

\section{Methodological considerations}

This study is the first to examine if fetal exposure to extra vitamin D may influence GDM development. The inclusion of all women from entire birth cohorts of the whole Danish population was made possible by the complete registration of every citizen via a CPR number into the Danish national health registers. By separating women from exposure groups by a specific point in time, selection bias was minimized. However, residual confounding cannot be excluded and in the following sections several potential confounders are discussed.

\section{Age at delivery}

Older women have a higher risk of developing GDM [24] and in this study we also saw a linear association between higher risk of GDM and older age at delivery. Age at delivery in our study was slightly higher in exposed compared to unexposed women, which advocated for a true association between the prenatal exposure to vitamin D fortification and GDM risk. However, women included in this study were relatively young and are not representative of all women that develop GDM.

\section{Trends in birth weight}

Increased birth weight has been associated with later onset of GDM [37]. However, no secular trend in birth weight between 1983 and 1988 were seen in this study (Online-Only Additional file 1: Table S6).

\section{Overweight and obesity}

The prevalence of overweight and obesity, which are strong risk factors of GDM [4], is increasing among women of childbearing age, particularly among young women [38]. Our sensitivity analyses that adjusted for pre-pregnancy BMI gave similar results as analyses without adjustment, suggesting that maternal BMI was not associated with the development of GDM in the present study. However, residual confounding related to fat mass or fat distribution, which we did not have information on, is still a possibility.

\section{Diet and vitamin $D$ supplementation}

No significant changes in margarine consumption in Denmark in the period analyzed have been reported [39]. Furthermore, there were no changes in recommendations regarding vitamin $D$ intake or vitamin supplementation during pregnancy between 1983 and 1988 [12]. Therefore, it is unlikely that these factors influenced our results.

\section{Bright sunshine hours as a source of vitamin $D$}

Sensitivity analyses including adjustment for individual bright sunshine hours showed similar results as the main analyses without adjustment, suggesting that the effect of the vitamin $\mathrm{D}$ fortification during gestation on later risk of GDM observed in this study was not confounded by vitamin $\mathrm{D}$ synthesized in the skin.

\section{Smoking}

We did not have data on smoking by the mothers of the women included, however, it is estimated that about $40 \%$ of all women smoked during pregnancy between 1983 and 1993 [40], and that the proportion of smokers, thus, did not differ between the exposed and unexposed group. On the other hand, the prevalence of women smoking during pregnancy has since been decreasing [41], and as smoking has been shown to be associated with GDM risk [42], such change in smoking habits might have influenced our results. However, sensitivity analyses adjusted for pregnancy smoking habits showed similar results as analyses without adjustment, suggesting that smoking during pregnancy did not confound the association observed.

\section{Assisted reproductive technology (ART)}

In Europe, the use of ART has been increasing over the years and an increased risk of GDM following ART treatment has been reported [43]. The chance that women conceived with the help of ART in our study was most likely slightly larger for unexposed than exposed women, and hence the use of ART could potentially have confounded the association observed. However, most women undergoing ART treatment in Denmark are over 30 years old and in the present study the oldest women were 27.5 years at time of delivery. Therefore, the risk of potential confounding by ART is expected to be minimal.

\section{Induced labor}

A more aggressive policy towards inducing delivery after $41+$ weeks was introduced in Denmark around 2009 [44], potentially leading the unexposed group to have less "time in pregnancy" to develop/or have a diagnosis of GDM. However, GDM is rarely diagnosed after term since routine screening is generally performed at the latest at 27-30 weeks of gestation [18]. When analyses were restricted to women delivering before 41 weeks of gestation, similar results were found (data not shown).

\section{GDM prevalence}

In Denmark, the prevalence of GDM is relatively low on a global scale and has increased from 1.7 to $2.9 \%$ between 2004 and 2012 [24]. Therefore, although the 
difference between the two exposure groups $(2.1 \%$ vs. $2.4 \%)$ may seem small, it might still be clinically relevant.

In this study, GDM risk increased with women's year of birth: from $2.0 \%$ in those born in 1983 to $2.9 \%$ in those born in 1988. This suggests that secular trends in GDM risk in Denmark may be present, making it difficult to disentangle the specific effects related to vitamin D fortification.

\section{GDM at subsequent pregnancies}

Women who develop GDM during their first pregnancy are at increased risk of developing GDM in their subsequent pregnancies [3]. Therefore, analyses to examine the risk of GDM in the second pregnancy, conditional on whether GDM was present in the first pregnancy, among women with two pregnancies, was planned. However, no women met the criteria.

\section{Diagnosis of GDM}

The diagnostic criteria for GDM did not change during the study period and by using the DMBR and DNPR, selection bias and risk of loss to follow-up could be avoided. Universal health coverage in Denmark secures that all Danish residents have access to healthcare regardless of their ethnic background or socioeconomic status. All deliveries, either at hospitals or at home with a midwife attending from the regional hospitals, are recorded in the registers. Therefore, most GDM diagnoses are registered in the DNPR.

Finally, we cannot exclude that other environmental and/or societal changes, such as an increase in the exposure to endocrine disrupting chemicals [45], may have influenced our results for instance through epigenetic changes influencing lifelong health and disease by modifying inflammatory molecular pathways and the immune response [46].

\section{Conclusion}

This study suggests that prenatal exposure to extra vitamin D from mandatory fortification may lower the risk of developing gestational diabetes among spring-born women, eg. from winter pregnancies, when the small extra amount of vitamin $D$ from fortification seemed particularly beneficial.

Our results may have public health relevance as they demonstrate that mothers consuming extra vitamin D from food fortification had daughters who were at lower subsequent risk of developing GDM. However, recommendations for vitamin D supplementation or fortification cannot be provided on the basis of this study and would require long-term randomized controlled trials.

\section{Additional file}

Additional file 1: In utero exposure to extra vitamin D from food fortification and the risk of subsequent development of gestational diabetes: the D-tect study. (DOCX 1837 kb)

\section{Acknowledgements \\ Not applicable. \\ Funding \\ The study was part of the 4-year project "D-tect" funded by the Programme Commission on Health, Food, and Welfare under the Danish Council for Strategic research (grant number 0603-00453B). The Lundbeck Foundation (grant number R170-2014-643). \\ The Danish Diabetes Academy supported by the Novo Nordisk Foundation. The funders had no role in the design of the study and collection, analysis, and interpretation of data and in writing the manuscript.}

\section{Availability of data and materials}

Access to the dataset may be granted upon request.

\section{Authors' contributions}

$\mathrm{BLH}$ designed the study. AK and BLH formulated the research question. RJ, PF, AV and PD provided feedback on the study design. AK analyzed the data. FT and PF provided methodological and statistical feedback. AK and MS wrote the manuscript. All authors read and provided written feedback on the manuscript. $\mathrm{BLH}$ is the guarantor of this work and, as such, had full access to all the data in the study and takes responsibility for the integrity of the data and the accuracy of the data analysis. All authors read and approved the final manuscript.

\section{Ethics approval and consent to participate}

The Danish Health Data Protection Agency (J.no. 2012-41-1156) provided permission to access the aforementioned registers. Approval from the ethical committee was not required.

\section{Consent for publication}

Not applicable.

\section{Competing interests}

The authors declare that they have no competing interests.

\section{Publisher's Note}

Springer Nature remains neutral with regard to jurisdictional claims in published maps and institutional affiliations.

\section{Author details}

${ }^{1}$ Research Unit for Dietary Studies at The Parker Institute Bispebjerg and Frederiksberg Hospital, part of the Copenhagen University Hospital - The capital Region, Nordre Fasanvej 57, vej 8, entrance 11, 2000 Frederiksberg, Denmark. ${ }^{2}$ Cardiovascular, Renal and Metabolic Disease (CVRM) Translational Medicine Unit, Early Clinical Development, IMED Biotech Unit, AstraZeneca, Gothenburg, Sweden. ${ }^{3}$ Center for Pregnant Women with Diabetes, Department of Obstetrics, Rigshospitalet, Copenhagen, Denmark. ${ }^{4}$ Institute of Clinical Medicine, Faculty of Health and Medical Sciences, University of Copenhagen, Copenhagen, Denmark. ${ }^{5}$ Research Unit for Chronic Conditions, Center of Clinical Research and Prevention, Bispebjerg og Frederiksberg Hospital, Capital Region, Frederiksberg, Denmark. ${ }^{6}$ The Boden Institute of Obesity, Nutrition, Exercise \& Eating Disorders, University of Sydney, Sydney, Australia. ${ }^{7}$ The National Institute of Public Health, University of Southern Denmark, Copenhagen, Denmark. ${ }^{8}$ The Department of Public Health, Section for General Medicine, University of Copenhagen, Copenhagen, Denmark.

Received: 16 May 2018 Accepted: 14 October 2018

Published online: 02 November 2018

\section{References}

1. Metzger BE, Buchanan TA, Coustan DR, et al. Summary and recommendations of the fifth international workshop-conference on gestational diabetes mellitus. Diabetes Care. 2007;30(Suppl 2):S251-60. https://doi.org/10.2337/dc07-s225. 
2. Damm P, Houshmand-Oeregaard A, Kelstrup L, et al. Gestational diabetes mellitus and long-term consequences for mother and offspring: a view from Denmark. Diabetologia. 2016;59:1396-9. https://doi.org/10.1007/ s00125-016-3985-5.

3. Getahun D, Fassett MJ, Jacobsen SJ. Gestational diabetes: risk of recurrence in subsequent pregnancies. Am J Obstet Gynecol. 2010;203:467.e1-6. https://doi.org/10.1016/j.ajog.2010.05.032.

4. Alzaim M, Wood RJ. Vitamin D and gestational diabetes mellitus. Nutr Rev. 2013;71:158-67. https://doi.org/10.1111/nure.12018.

5. Moses RG, Brand-Miller JC. Dietary risk factors for gestational diabetes mellitus: are sugar-sweetened soft drinks culpable or guilty by association? Diabetes Care. 2009;32:2314-5. https://doi.org/10.2337/dc09-1640.

6. Zhang C, Schulze MB, Solomon CG, Hu FB. A prospective study of dietary patterns, meat intake and the risk of gestational diabetes mellitus. Diabetologia. 2006;49:2604-13. https://doi.org/10.1007/s00125-006-0422-1.

7. Zhang M-X, Pan G-T, Guo J-F, et al. Vitamin D deficiency increases the risk of gestational diabetes mellitus: a meta-analysis of observational studies. Nutrients. 2015;7:8366-75. https://doi.org/10.3390/nu7105398.

8. De-Regil LM, Palacios C, Lombardo LK, Peña-Rosas JP. Vitamin D supplementation for women during pregnancy. Cochrane Database Syst Rev. 2016;(1):CD008873. https://doi.org/10.1002/14651858.CD008873.pub3.

9. Zhang $Y$, Gong $Y, X u e ~ H$, et al. Vitamin $D$ and gestational diabetes mellitus: a systematic review based on data free of Hawthorne effect. BJOG An Int J Obstet Gynaecol. 2018;125:784-93. https://doi.org/10.1111/1471-0528.15060.

10. Joergensen JS, Lamont RF, Torloni MR. Vitamin D and gestational diabetes. Curr Opin Clin Nutr Metab Care. 2014;17:360-7. https://doi.org/10.1097/ MCO.0000000000000064.

11. Jacobsen R, Frederiksen P, Heitmann BL. Exposure to sunshine early in life prevented development of type 1 diabetes in Danish boys. J Pediatr Endocrinol Metab. 2016;29:417-24. https://doi.org/10.1515/jpem-2015-0393.

12. Stougaard $M$, Damm $P$, Frederiksen $P$, et al. Exposure to vitamin $D$ from fortified margarine during fetal life and later risk of pre-eclampsia: the Dtect study. Public Health Nutr. 2018;21(4):721-31. https://doi.org/10.1017/ S1368980017003135

13. Minister of Agriculture and Fishing (1937) Ministerial order no. 247 28/7 on the effect of vitamins in margarine in the Danish law gazette.

14. Keller A, Ängquist L, Jacobsen $R$, et al. A retrospective analysis of a societal experiment among the Danish population suggests that exposure to extra doses of vitamin a during fetal development may lower type 2 diabetes mellitus (T2DM) risk later in life. Br J Nutr. 2017;117:731-6. https://doi.org/10. 1017/S000711451700037X.

15. Haraldsdóttir J, Holm L, Jensen J, Møller A (1985) Danish Dietary Habits 1985. The main results [In Danish: Danskernes kostvaner 1985. Hovedresultater].

16. Knudsen LB, Olsen J. The Danish medical birth registry. Dan Med Bull. 1998:45:320-3.

17. Lynge E, Sandegaard JL, Rebolj M. The Danish National Patient Register. ScandJPublic Heal. 2011;39:30-3. https://doi.org/10.1177/1403494811401482.

18. Jensen DM, Mølsted-Pedersen L, Beck-Nielsen $H$, et al. Screening for gestational diabetes mellitus by a model based on risk indicators: a prospective study. Am J Obstet Gynecol. 2003;189:1383-8.

19. Araujo Júnior E, Peixoto AB, Zamarian ACP, et al. Macrosomia. Best Pract Res Clin Obstet Gynaecol. 2017;38:83-96. https://doi.org/10.1016/j.bpobgyn.2016.08.003.

20. Marsal K, Persson $P$, Larsen $T$, et al. Intrauterine growth curves based on ultrasonically estimated foetal weights. Acta Paediatr. 1996;85:843-8.

21. Hyppönen $E_{1}$ Power $C$. Hypovitaminosis $D$ in British adults at age $45 \mathrm{y}$ : nationwide cohort study of dietary and lifestyle predictors. Am J Clin Nutr. 2007;85:860-8.

22. Textor J, Hardt J, Knüppel S. DAGitty: a graphical tool for analyzing causal diagrams. Epidemiology. 2011;22:745. https://doi.org/10.1097/EDE. 0b013e318225c2be.

23. Cole SR, Platt RW, Schisterman EF, et al. Illustrating bias due to conditioning on a collider. Int J Epidemiol. 2010;39:417-20. https:/doi.org/10.1093/ije/dyp334.

24. Jeppesen C, Maindal HT, Kristensen JK, et al. National study of the prevalence of gestational diabetes mellitus among Danish women from 2004 to 2012. Scand J Public Health. 2017;45(8):811-7. https://doi.org/10. 1177/1403494817736943.

25. Cappelen J, Kern-Hansen C, Laursen EV, et al. Denmark -DMI Historical Climate Data Collection. 2012. p. 1768-2011

26. Krishnaveni GV, Veena SR, Winder NR, et al. Maternal vitamin D status during pregnancy and body composition and cardiovascular risk markers in Indian children: the Mysore Parthenon study. Am J Clin Nutr. 2011;93:628-35. https://doi.org/10.3945/ajcn.110.003921.
27. Nguyen QM, Srinivasan SR, Xu J-H, et al. Utility of childhood glucose homeostasis variables in predicting adult diabetes and related Cardiometabolic risk factors: the Bogalusa heart study. Diabetes Care. 2010; 33:670-5. https://doi.org/10.2337/dc09-1635.

28. Zipitis CS, Akobeng AK. Vitamin D supplementation in early childhood and risk of type 1 diabetes: a systematic review and meta-analysis. Arch Dis Child. 2008;93:512-7. https://doi.org/10.1136/adc.2007.128579.

29. Jacobsen R, Hypponen E, Sørensen TIA, et al. Gestational and early infancy exposure to margarine fortified with vitamin D through a National Danish Programme and the risk of type 1 diabetes: the D-Tect study. PLoS One. 2015;10:e0128631. https://doi.org/10.1371/journal.pone.0128631.

30. Nascimento FAM, Ceciliano TC, Aguila MB, Mandarim-de-Lacerda CA. Transgenerational effects on the liver and pancreas resulting from maternal vitamin D restriction in mice. J Nutr Sci Vitaminol (Tokyo). 2013;59:367-74.

31. Javaid M, Crozier S, Harvey $\mathrm{N}$, et al. Maternal vitamin D status during pregnancy and childhood bone mass at age 9 years: a longitudinal study. Lancet. 2006;367:36-43. https://doi.org/10.1016/S0140-6736(06)67922-1.

32. Hales CN, Barker DJ. Type 2 (non-insulin-dependent) diabetes mellitus: the thrifty phenotype hypothesis. Diabetologia. 1992;35:595-601.

33. Cleal JK, Day PE, Simner CL, et al. Placental amino acid transport may be regulated by maternal vitamin $\mathrm{D}$ and vitamin D-binding protein: results from the Southampton Women's survey. Br J Nutr. 2015;113:1903-10. https://doi.org/10.1017/S0007114515001178.

34. Kumar PU, Ramalaxmi BA, Venkiah K, Sesikeran B. Effect of maternal undernutrition on human foetal pancreas morphology in second trimester of pregnancy. Indian J Med Res. 2013;137:302-7.

35. Omu EA. Pro-inflammatory cytokines, lipid metabolism and inflammation in gestational diabetes mellitus as cause of insulin resistance. Chapter from the book Gestational Diabetes - Causes, Diagnosis and Treatment. 2013:79-102. https://doi.org/10.5772/55634.

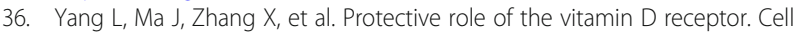
Immunol. 2012;279:160-6. https://doi.org/10.1016/j.cellimm.2012.10.002.

37. Innes KE, Byers TE, Marshall JA, et al. Association of a Woman's own birth weight with subsequent risk for gestational diabetes. JAMA. 2002;287:2534. https://doi.org/10.1001/jama.287.19.2534.

38. Ovesen P, Rasmussen S, Kesmodel U. Effect of Prepregnancy maternal overweight and obesity on pregnancy outcome. Obstet Gynecol. 2011;118: 305-12. https://doi.org/10.1097/AOG.0b013e3182245d49.

39. Forsyningen af fødevarer 1955-1999: Udviklingen i danskernes kost forbrug, indkøb og vaner - Translation: The supply of food 1955-1999: Developments in the Danish diet - consumption, purchasing and habits.

40. Osler M. Smoking habits in Denmark from 1953 to 1991: a comparative analysis of results from three nationwide health surveys among adult Danes in 1953-1954, 1986-1987 and 1990-1991. Int J Epidemiol. 1992;21:862-71.

41. Egebjerg Jensen K, Jensen A, Nøhr B, Krüger Kjær S. Do pregnant women still smoke? A study of smoking patterns among 261,029 primiparous women in Denmark 1997-2005. Acta Obstet Gynecol Scand. 2008;87:760-7. https://doi.org/10.1080/00016340802179814.

42. Dodds L, Woolcott CG, Weiler H, et al. Vitamin D status and gestational diabetes: effect of smoking status during pregnancy. Paediatr Perinat Epidemiol. 2016;30:229-37. https://doi.org/10.1111/ppe.12278.

43. Holst S, Kjær SK, Jørgensen ME, et al. Fertility problems and risk of gestational diabetes mellitus: a nationwide cohort study. Fertil Steril. 2016; 106:427-434.e1. https://doi.org/10.1016/j.fertnstert.2016.03.040.

44. Hedegaard M, Lidegaard $\varnothing$, Skovlund $C$, et al. Perinatal outcomes following an earlier post-term labour induction policy: a historical cohort study. BJOG An Int J Obstet Gynaecol. 2015;122:1377-85. https://doi.org/10.1111/1471-0528.13299.

45. Ehrlich S, Lambers D, Baccarelli A, et al. Endocrine disruptors: a potential risk factor for gestational diabetes mellitus. Am J Perinatol. 2016;33:1313-8. https://doi.org/10.1055/s-0036-1586500.

46. Indrio F, Martini S, Francavilla $\mathrm{R}$, et al. Epigenetic matters: the link between early nutrition, microbiome, and long-term health development. Front Pediatr. 2017;5:178. https://doi.org/10.3389/fped.2017.00178. 